CASO CLÍNICO

\title{
Dos casos de reemplazo transcatéter de válvula aórtica en Honduras, año 2019
}

\author{
Two cases of aortic valve transcateter replacement in Honduras, 2019
}

Ramón Alberto Martínez Schweinfurth, ${ }^{1}$ Haroldo López, ${ }^{2}$ Karen Melissa Sánchez Barjun. ${ }^{1}$

${ }^{1}$ Médico Especialista en Medicina Interna; Hospital CEMESA; Hospital Dr. Mario Catarino Rivas; San Pedro Sula; ${ }^{2} \mathrm{MD}, \mathrm{PhD}$ en Hemodinamia y Cardiología Intervencionista; Hospital Medical Center; Tegucigalpa; Honduras.

RESUMEN. Antecedentes: Las valvulopatías cardiacas se presentan en 1 de 100,000 habitantes en Centro América. La estenosis aórtica representa el $25 \%$ de estas patologías. La principal causa es la fiebre reumática y en países de ingresos altos, las anomalías congénitas. La valvulopatía aórtica bicúspide es una anomalía rara, presente en $0.5 \%$ de la población total. Tradicionalmente en Honduras, las correcciones de estas anomalías se habían hecho con cirugía vascular. Descripción de los casos clínicos: Se presentan los dos primeros casos realizados en Honduras mediante reemplazo transcateter 0 terapia endovascular (transcatheter aortic valve replacement, TAVR), en el año 2019, en un centro asistencial privado en Tegucigalpa. El primer caso sobre una aorta bivalva, con el apoyo de un sistema En Snare, por el difícil abordaje, con EuroScore II $<4 \%$. El segundo caso sobre una estenosis aórtica secundaria a fiebre reumática. Ambos procedimientos se ejecutaron sin complicaciones inmediatas o tardías. La ecocardiografía reportó resolución de los gradientes de presión y reducción de la velocidad transvalvular máxima aórtica (Vmax Ao). Conclusión: Siendo esta una terapia de primer mundo, respaldada para pacientes de alto y bajo riesgo quirúrgico, abre la oportunidad a nuestra población de recibir una terapia efectiva, segura y con resultados inmediatos.

Palabras Clave. Aorta, Enfermedades de la aorta, Estenosis de la válvula aórtica, Reemplazo de la válvula aórtica transcatéter.

Recibido: 25-09-2019 Aceptado 20-01-2021 Primera vez publicado en linea 16-03-2021 Dirigir correspondencia a: Dr. Ramón Alberto Martínez Schweinfurth

Correo electrónico: rmartinez.scu@gmail.com,

Declaración de relaciones y actividades financieras y no financieras y conflictos de interés: ninguno.

DOl: https://doi.org/10.5377/rmh.v89i1.11585

(C) 2021 Autor(es): (c) (i)

\section{INTRODUCCIÓN}

Las valvulopatías cardíacas se presentan en 1 de cada 100,000 habitantes en países centroamericanos. La fiebre reumática es la causa principal en países de bajos y medianos ingresos (65\% de los casos). En países de altos ingresos predominan cuadros degenerativos o inflamatorios que culminan en engrosamiento, calcificación, y disfunción de las válvulas. ${ }^{1}$

La estenosis aórtica (EA), está presente en el $25 \%$ de los pacientes con alguna valvulopatía cardíaca. Es la valvulopatía principal que conlleva intervenciones quirúrgicas. ${ }^{2}$ Se ha documentado válvulas aórticas bicúspides en 0.5 a $1.4 \%$ de la población general. ${ }^{2}$ Otras causas son las valvulopatías calcificadas degenerativas, fiebre reumática y radiación. ${ }^{1,3}$ Causas menos comunes incluyen las que se presentan en jóvenes con hiperlipoproteinemia tipo II, en quienes ocurre engrosamiento de las valvas de forma nodular por depósito de colesterol (similar al proceso de aterosclerosis). ${ }^{3}$

El proceso de calcificación de la válvula aórtica, es progresivo. Inicia con la activación del endotelio, luego, hay acúmulo de lípidos, liberación de células inflamatorias, con ellas, citosinas, y activación de miofibroblastos. Estos generan matriz ósea, y se diferencian fenotípicamente en osteoblastos. Crean y depositan hidroxiapatita cálcica, y se da el proceso de calcificación valvular. Cuando la obstrucción se presenta desde el nacimiento, el gradiente tensional entre el ventrículo izquierdo (VI) y la aorta, se conserva relativamente, aumenta poco a poco, conservando la función contráctil del VI, gracias a la hipertrofia concéntrica de éste. A lo largo del tiempo, la hipertrofia excesiva, se vuelve un proceso patológico, ya que inicia el proceso de fibrosis del miocardio y la disfunción diastólica de este. ${ }^{1}$ Mientras el miocardio logre sostener el gasto cardiaco, el paciente permanecerá asintomático. Sin embargo, llegará el momento donde el miocardio no podrá sostener el gasto según la demanda con el ejercicio, y posteriormente, iniciaran los síntomas típicos de EA. Esto, se presenta usualmente cuando el orificio valvular es menor a $1 \mathrm{~cm}^{2}$. Hay 4 categorías para definir EA (ver Cuadro 1). Se considera como lesión severa: jet aórtico $>4 \mathrm{~m} / \mathrm{seg}$; un gradiente de presión sistólica $>40 \mathrm{mmHg}$ con un gasto cardiaco normal y área valvular $<1 \mathrm{~cm}^{2}$. La sintomatología usual es disnea a los esfuerzos, angina y síncope. La disnea 
Cuadro 1. Clasificación de la estenosis aórtica. ${ }^{2}$

\begin{tabular}{|c|c|}
\hline Clasificación & Hallazgo Ecocardiográfico \\
\hline (EA) de gradiente elevado & $\begin{array}{l}\mathrm{AV}<1 \mathrm{~cm}^{2} \\
\text { Gradiente Aórtico }>40 \mathrm{mmHg}\end{array}$ \\
\hline $\begin{array}{l}\text { EA de bajo flujo, bajo gradiente y FEVi } \\
\text { reducida }\end{array}$ & $\begin{array}{l}\text { AV }<1 \mathrm{~cm}^{2} \\
\text { Gradiente Aórtico }<40 \mathrm{mmHg} \\
\mathrm{FEVi}<50 \% \\
\text { Volumen de eyección } \leq 35 \mathrm{ml} / \mathrm{m}^{2}\end{array}$ \\
\hline $\begin{array}{l}\text { EA de bajo flujo, bajo gradiente y FEVi } \\
\text { conservada }\end{array}$ & $\begin{array}{l}\text { AV }<1 \mathrm{~cm}^{2} \\
\text { Gradiente Aórtico }<40 \mathrm{mmHg} \\
\mathrm{FEVi} \geq 50 \% \\
\text { Volumen de eyección } \leq 35 \mathrm{ml} / \mathrm{m}^{2}\end{array}$ \\
\hline $\begin{array}{l}\text { EA de flujo normal, bajo gradiente y FEVi } \\
\text { conservada }\end{array}$ & $\begin{array}{l}\mathrm{AV}<1 \mathrm{~cm}^{2} \\
\text { Gradiente aórtico }<40 \mathrm{mmHg} \\
\mathrm{FEVi} \geq 50 \% \\
\text { Volumen de eyección }>35 \mathrm{ml} / \mathrm{m}^{2}\end{array}$ \\
\hline
\end{tabular}

EA: estenosis aórtica; AV: área valvular; FEVi: Fracción de eyección del ventrículo izquierdo.

se presenta por el incremento de la tensión capilar pulmonar, secundaria a la presión diastólica elevada del VI. La angina es por desequilibrio entre la demanda y el aporte de oxígeno del mismo miocardio. El síncope es causado por vasodilatación de los músculos que se ejercitan y vasoconstricción inadecuada de los músculos en reposo. Esto último, en un corazón que no puede aumentar el gasto cardiaco. ${ }^{1}$

El estudio ecocardiográfico es el de elección ya que permite evaluar el grado de estenosis mediante la modalidad doppler, la función ventricular, el engrosamiento del miocardio y documentar otras patologías aórticas concomintantes. ${ }^{2}$ Estudios ecocardiográficos en poblaciones de 65 años o más han encontrado hasta un $2 \%$ de prevalencia. En los pacientes asintomáticos se recomienda realizar pruebas de esfuerzo para poder desenmascarar los síntomas y estratificar el riesgo que conlleva el paciente. Esto se realiza mediante ecocardiografia, evaluando los cambios adaptativos del VI según la demanda o la ausencia de esto, sin generar riesgo al paciente durante el procedimiento. ${ }^{2,4}$ La ecocardiografía transesofágica tiene gran relevancia previo al reemplazo valvular, permitiendo mayor detalle sobre el estado de la válvula mitral. ${ }^{5}$ Sin embargo, a todo paciente con plan de reemplazo valvular, se le debe realizar una tomografía computarizada multicortes, ya que esto permite evaluar las dimensiones de la raíz aórtica, forma y tamaño de la válvula aórtica, la distancia entre el nacimiento de las arterias coronarias y el anillo valvular, el nivel de calcificación y la tortuosidad de los vasos. La resonancia magnética es una opción; sin embargo, la tomografía sigue siendo superior a esta. ${ }^{2}$

La primera prótesis endovascular realizada en un humano adulto fue realizada en el año 2002 por Alain Cribier y colaboradores. ${ }^{5}$ Esto fue realizado en un varón de 57 años de edad con EA calcificada en shock cardiogénico. Esto fue realizado abordando la Vena femoral derecha y llegando al anillo valvular aortico mediante punción transeptal. Posteriormente se propuso el abordaje transapical (mediante una incisión torácica, se expone el ápex del corazón y se punciona con un catéter $7 \mathrm{Fr}$, para introducir la válvula y dirigirla a su destino) en la cual introducen la aplicación de marcapaso transitorio para generar taquicardia
(160-220 lpm) mientras se entrega la prótesis valvular, evitar el reto de puncionar el septum, y el avance tortuoso a través del VI, luego la válvula mitral, siendo esto causa de insuficiencia severa. ${ }^{6-7}$ Luego se crearon válvulas auto-expandibles que permitían auto centrado y cierto grado de reposicionamiento. ${ }^{7,8}$ La CoreValve ${ }^{\mathrm{TM}}$, consiste en tejido pericárdico de origen bovino, montado en una malla metálica de nitinol autoexpandible (ver Figura 1). Diseñado con alta fuerza radial en la parte baja que permite desplazar las valvas calcificadas, la parte media, especialmente diseñada para evitar las coronarias, y la parte superior para ajustarse a la aorta. Esta prótesis se entrega vía retrograda, con abordaje inguinal. ${ }^{8}$

A continuación, se presentan dos casos de EA sometidos a reemplazo valvular transcateter o terapia endovascular (transcatheter aortic valve replacement, TAVR). Un paciente con aorta bivalva y otro con EA secundaria a cambios escleróticos. Los casos fueron manejados en un centro asistencial privado en Tegucigalpa en el año 2019, constituyéndose en los primeros casos de TAVR publicados en Honduras.

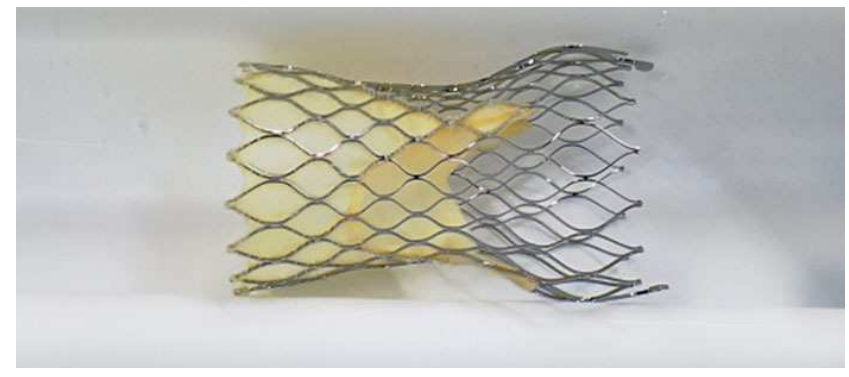

Figura 1. Imagen de una prótesis aórtica auto-expandible de nitinol. Este tipo de prótesis fue utilizado en ambos casos informados en el presente artículo (fotografía realizada previo al procedimiento).

\section{DESCRIPCIÓN DE LOS CASOS}

\section{Caso 1}

Paciente masculino de 68 años. Conocido por ser hipertenso hace 12 años, tratado con bisoprolol $5 \mathrm{mg}$ y candesartan $16 \mathrm{mg}$ al día. Hipertrigliceridemia, tratado con fenofibrato $10 \mathrm{mg}$ vía oral al día. Fue diagnosticado con aorta bivalva desde su juventud. Sin plan quirúrgico, ni seguimiento hasta la adultez. Negó tabaquismo y refirió consumo ocasional de alcohol. Con estilo de vida sedentario. Quien 6 meses previo a su implante, fue programado para realizar artroscopía izquierda, sufre sincope minutos previo a la inducción de la anestesia por lo que es enviado al servicio de cardiología para revaloración. Encontrándole con tensión arterial de: $118 / 72 \mathrm{mmHg}$, midiendo $1.69 \mathrm{~m}$ de altura y pensando $75 \mathrm{~kg}$, brindando un IMC: $26.7 \mathrm{~kg} / \mathrm{m}^{2}$. Se documenta EA severa por Ecocardiografia Transtorácica, y se procede con un ecocardiograma transesofágico (ver Cuadro 2). Debido a los hallazgos ecocardiográficos se decide someter a TAVR.

El abordaje fue transfemoral derecha. Con técnica convencional de Seldinger y arterio-disección para la introducción de la prótesis valvular. Se acompañó con la introducción de un marcapaso transitorio y la introducción de ecocardiografía transeso- 
Cuadro 2. Hallazgos ecocardiográficos previos y posterior a la colocación de prótesis valvular, dos casos clínicos, Tegucigalpa, 2018.

\begin{tabular}{lcccc}
\hline DESCRIPCION & \multicolumn{2}{c}{ CASO 1 } & \multicolumn{2}{c}{ CASO 2 } \\
& EPI & EC & EPI & EC \\
\hline Raíz Aortica $(\mathrm{mm})$ & 40 & 40 & 35 & 32 \\
Al $(\mathrm{mm})$ & 40 & 40 & 40 & 42 \\
DTSVI $(\mathrm{mm})$ & 15 & 14 & 16 & 15 \\
TIV $(\mathrm{mm})$ & 15 & 12 & 16 & 16 \\
DTDVI $(\mathrm{mm})$ & 45 & 46 & 40 & 40 \\
FEVI $(\%)$ & 65 & 69 & 63 & $>70$ \\
Vmax Ao $(\mathrm{m} / \mathrm{s})$ & 4.6 & 2.5 & 4.5 & 1.5 \\
Vmax TSVI $(\mathrm{m} / \mathrm{s})$ & 1.04 & 1.2 & 1.2 & 1.4 \\
E $(\mathrm{m} / \mathrm{s})$ & 0.66 & 0.75 & 0.65 & 0.5 \\
A $(\mathrm{m} / \mathrm{s})$ & 0.87 & 0.99 & 1.07 & 0.99 \\
E/A & 0.75 & 0.76 & 0.610 & 0.51 \\
\hline
\end{tabular}

EPI=ecocardiografía previa intervención; EC=ecocardiografía control (postintervención)

Al: aurícula izquierda; DTSVI: diámetro telesistólico del ventrículo izquierdo; TIV: tabique interventricular; DTDVI: diámetro telediastólico del ventrículo izquierdo; FEVI: fracción de eyección del ventrículo izquierdo; Vmax Ao: velocidad máxima válvula aórtica; Vmax TSVI: velocidad máxima en tracto de salida. $\mathrm{E}$ : onda $\mathrm{E} ; \mathrm{A}$ : onda $\mathrm{A}$.

fágica. Entre las dificultades técnicas, la angulación de la aorta con el plano valvular aórtico no permitía el cruce de la valvular percutánea. Por ello, se apoyó con una segunda guía femoral contralateral, enlazando la guía de la válvula con un sistema En Snare al momento de implantarla (ver Figura 2). Esto con el objetivo de lograr el cruce de la aorta. El ecocardiograma control (ver Cuadro 2), mostró buena contractilidad global y segmentaria del VI. Sin trombos ni masas intracavitarias. La prótesis en adecuada posición, sobresaliendo aproximadamente 2 $\mathrm{mm}$ hacia el tracto de salida del Ventrículo Izquierdo (TSVI). Sin trombos y con movimiento normal de sus cúspides. Aumento de las velocidades transaórticas y presencia de un ligero flujo perivalvular en vecindad con el septum interventricular. Clínicamente, sin presentar embolismos cerebrales y con resolución de la disnea y sincope.

\section{Caso 2}

Masculino de 63 años, sin patologías conocidas de base. Sufre de obesidad mórbida en los últimos 10 años. El hermano es cardiópata (cardiopatía isquémica), a quien se le hizo angioplastía. Se presentó con cuadro de disnea a los grandes esfuerzos que progresó a los pequeños esfuerzos. El último mes, ya con disnea en reposo. Al examinarle con tensión arterial de 150/86 mmHg, Frecuencia Cardiaca 88 latidos por minuto, 18 respiraciones por minuto, en reposo. Afebril. Con $1.74 \mathrm{~m}$ de altura, $136 \mathrm{~kg}$ de peso e IMC $45 \mathrm{Kg} / \mathrm{m}^{2}$. Se le realizó ecocardiograma como parte de su evaluación. Debido a los hallazgos compatibles con EA severa y se decide programar para TAVR.

El abordaje fue femoral izquierda, por punción y técnica de Seldinger y luego arterio-disección. Se realizaron bajo anestesia general, con intubación endotraqueal y realización de ecocardiograma transesofágico durante la intervención. Se introdujo catéter guía a través del cual se guió la prótesis. Centrándola y entregándola en la válvula aórtica, sin complicaciones (ver Figura 3).
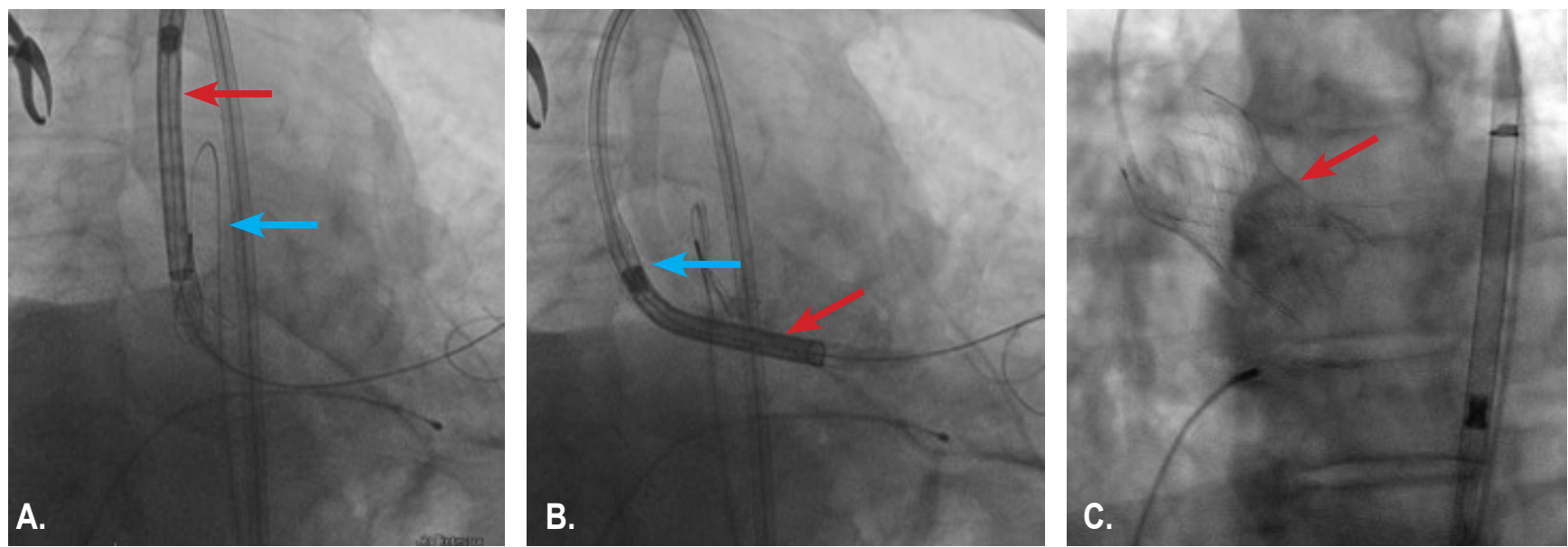

Figura 2. Prótesis aórtica implantada guiada por En Snare System (Caso 1). Imágenes tomadas en el transoperatorio. (A) Prótesis endovascular siendo redirigida por el sistema En Snare, para poder introducirla a través del anillo valvular. (B) Prótesis valvular ubicada a través del anillo valvular con el sistema En Snare. (C) Previo retiro del sistema En Snare, liberación de la prótesis en el anillo valvular aórtico. Flecha azul: sistema En Snare; Flecha Roja: prótesis aórtica auto-expandible de nitinol. 


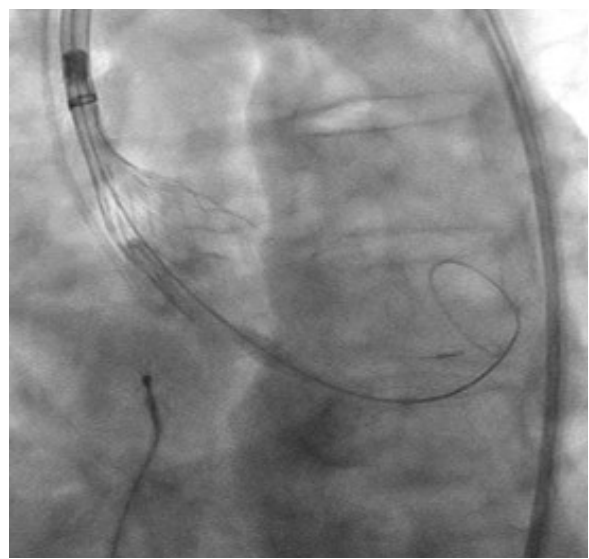

Figura 3. Liberación de prótesis aórtica transcatéter (Caso 2). Imagen tomada en el transoperatorio. Se aprecia la liberación de la endoprótesis en el anillo valvular aórtico.

\section{DISCUSIÓN}

El manejo farmacológico de la EA es controversial. Los pacientes se pueden beneficiar de diuréticos cuando hay retención de líquidos. Sin embargo, esto se debe vigilar con cautela, ya que depletar al paciente de volumen compromete el volumen diastólico, generando disminución del gasto cardiaco, llevando a hipotensión ortostática. Los Inhibidores de la Enzima Convertidora de Angiotensina (IECA's), se utilizan en pacientes que no son candidatos para cirugía, a dosis bajas. Los betabloqueantes, están contraindicados por la depresión del miocardio que generan. ${ }^{2}$ Por tal motivo, el cambio valvular es la terapia ideal y definitiva.

Las indicaciones para corrección de estenosis valvular aórtica son a) pacientes sintomáticos, con estenosis severa y gradiente elevado (lb y b) pacientes sintomáticos con bajo flujo, bajo gradiente, fracción de eyección disminuida, pero con reserva cardiaca (IC). ${ }^{2}$ También en pacientes asintomáticos con EA severa y disfunción sistólica del VI $(\mathrm{FEVi}<50 \%)$, sin otra etiología, y pacientes con EA severa con una prueba de esfuerzo positiva claramente justificada por la EA. Nuestros pacientes se incluyen bajo la clasificación de EA de gradiente elevado. Siendo ambos sintomáticos. Razón de la indicación de TAVR.

Las intervenciones deben realizarse a la mayor brevedad posible. Independientemente del valor de FEVi, y siempre que se mantenga un gradiente $>40 \mathrm{mmHg}$. ${ }^{2}$ Se ha documentado mejoría en pacientes con gradientes menores; sin embargo, hay mayor mortalidad en aquellos con gradientes $\leq 20 \mathrm{mmHg}{ }^{9 \cdot 11}$ Es importante mencionar, que en pacientes con una sobrevida $<1$ año, con enfermedades concomitantes severas y/o edad muy avanzada que evite generar una mejoría en la calidad de vida, no son candidatos para ser intervenidos. ${ }^{2}$ Ambos pacientes seleccionados, eran menores de 70 años. Las patologías eran hipertensión arterial y dislipidemias, que no contraindican ni cambian el pronóstico del paciente, ya que no son los causantes de la sintomatología.

La elección entre un procedimiento abierto o endovascular depende del riesgo del paciente. Si este es clasificado con bajo riesgo quirúrgico (EuroScore $\|<4 \%$ o logistic EuroScore $\mid<10 \%$ ) y no se trate de un paciente fiable, sufra de aorta en porcelana, o sea secundario a radioterapia torácica, puede ser sometido a cirugía cardiaca abierta. Esto deja a la TAVR, para el paciente sin opción quirúrgica por alto riesgo (EuroScore $\| \geq 4 \%$ o logistic EuroScore $\mathrm{I} \geq 10 \%$ ) y en adultos mayores con buen abordaje transfemoral. ${ }^{2}$ Estudios como el SURTAVI, multicéntrico, donde aleatorizaron 1746 pacientes, con edades promedio de 79 años, clasificados con riesgo quirúrgico intermedio (Society of Thoracic Surgeons Predicted Risk of Mortality), ${ }^{12}$ mostraron que la TAVR no es inferior a la intervención quirúrgica en pacientes con este riesgo quirúrgico, además, las complicaciones como eventos vasculocerebrales (EVC) y la calidad de vida mejoraron con TAVR.${ }^{13}$ Haciendo esta terapia más popular en los Estados Unidos el día de hoy. ${ }^{14}$ Sin embargo, recientemente fue publicado el "Evolut low Risk Trail."15 Estudio en el cual, compararon TAVR e intervención quirúrgica en pacientes con bajo riesgo quirúrgico. De un total de 1403 pacientes, con edad media de 74 años, encontraron mayor sobrevida a los 24 meses, EVC leves, menores sangrados, lesión renal y fibrilación auricular a los 30 dias en el grupo de TAVR. ${ }^{15}$ Lo que abre la ventana para los pacientes de bajo riesgo. Siendo el primero de los casos presentados de bajo riesgo (Euroscore II 0.98\%). Además, este caso corresponde a una válvula biscupide, haciéndolo un caso raro, ya que se presenta en 0.5 a $1.4 \%$ de la población. Por consiguiente, son pocos los casos documentados a los cuales se le han intervenido y realizado implantes endovasculares. Debido a la anatomía del paciente, se vio en la obligación de utilizar una segunda guía y un sistema En Snare al momento del implante. Dicha técnica, no descrita en el proceso de TAVR.

Actualmente, se ha dado apertura para el uso de TAVR en pacientes de bajo y alto riesgo quirúrgico. Lo cual, abre campo a la realización de un mayor número de procedimientos. Además, brinda la oportunidad de ofrecer un método más seguro que los métodos quirúrgicos tradicionales. Estos dos procedimientos, abren camino a la realización de un número mayor de casos. Además, documenta la posibilidad de utilizar maniobras avanzadas como el uso de un sistema En Snare, para poder redirigir y resolver cualquier dificultad en el abordaje y liberación de prótesis en el anillo valvular en nuestro país. Honduras se une a la medicina de primer mundo con estos procedimientos.

\section{CONTRIBUCIONES}

Todos los autores concibieron la preparación del caso clínico. RAM y KMS lideraron la redacción. $\mathrm{HL}$, médico tratante, brindó acceso a la información clínica de los casos y oriento la estructura del documento. Todos los autores aprobaron la versión final del artículo, así como aprobaron la versión revisada incorporando las recomendaciones editoriales.

\section{AGRADECIMIENTOS}

Agradecemos a la Dirección Médica del Hospital Medical Center, Tegucigalpa, por facilitarnos el acceso a los registros clínicos de los casos.

\section{DETALLES DE AUTORES}

${ }^{1}$ Ramón A. Martínez Schweinfurth rmartinez.scu@gmail.com ${ }^{2}$ Haroldo López

${ }^{1}$ Karen Melissa Sánchez Barjun haroldologar@yahoo.com karen_barjun@hotmail.com 


\section{REFERENCIAS}

1. O'Gara PT, Loscalzo J. Valvulopatía aórtica. En: Kasper DL, Fauci AS, Hauser SL, Longo DL, Jamelson JL, Loscalzo JL, Editores Harrison Principios de Medicina Interna. 19a ed. México: McGrawHill; 2015. p.15281534.

2. Baumgartner H, Falk V, Bax JJ, De Bonis M, Hamm C, Holm PJ. 2017 ESC/EACTS Guidelines for the management of valvular heart disease. Eur Heart J. 2017; 38: 2739-2791.

3. Otto CM, Bonow RO. Valvular heart disease. En: Bonow RO, Mann D, Zipes D, Libby P Braunwald's heart disease: A Textbook of Cardiovascular Medicine 9a ed. Philadelphia: Saunders; 2012. p.1468-1539.

4. Rafique AM, Biner S, Ray I, Forrester JS, Tolstrup K, Siegel RJ. MetaAnalysis of Prognostic Value of Stress Testing in Patients with Asymptomatic Severe Aortic Stenosis. Am J Cardiol. 2009; 104(7):972-977.

5. Cribier A, Eltchaninoff H, Bash A, Borenstein N, Tron C, Bauer F, et al. Percutaneous transcatheter implantation of an Aortic Valve Prothesis of Calcific Aortic Stenosis, first human case description. Circulation. 2002. 106(24): 3006-3008.

6. Lichtenstein SV, Cheung A, Ye J, Thompson CR, Carere RG, Pasupati S, et al. Transapical transcatheter aortic valve implantation in humans, initial clinical experience. Circulation. 2006. 114(6): 591-596.

7. Zamorano JL, Badano LP, Bruce C, Chan KL, Goncalves A, Hahn RT, et al. EAE/ASE recommendations for the use of echocardiography in new transcatheter interventions for valvular heart disease. Eur Heart J. 2001: 32(17): 2189-2214

8. Grube E, Laborde JC, Zickmann B, Gerckens U, Felderhoff T, Sauren B, et al. First report on a human percutaneous transluminal implantation of a self-expanding valve prosthesis for interventional treatment of aortic valve stenosis. Catheter Cardiovasc Interv. 2005;66:465-9.

9. Monin JL, Quéré JP, Monchi M, Petit H, Baleynaud S, Chauvel C; et al. Low-gradient aortic stenosis: operative risk stratification and predictors for long-term outcome: a multicenter study using dobutamine stress hemodynamics. Circulation. 2003;108(3):319-324.

10. Tribouilloy $C$, Levy $F$, Rusinaru $D$, Guéret $P$, Petit-Eisenmann $H$, Baleynaud $\mathrm{S}$, et al. Outcome after aortic valve replacement for low-flow/ low-gradient aortic stenosis without contractile reserve on dobutamine stress echocardiography. J Am Coll Cardiol. 2009;53(20):1865-1873.

11. Levy F, Laurent M, Monin JL, Maillet JM, Pasquet A, Le Tourneau T, et al. Aortic valve replacement for low-flow / low gradient Aortic Stenosis. J Am Coll Cardiol. 2008;51(15):1466-72.

12. Reardon MJ, Van Mieghem NM, Popma JJ, Kleiman NS, Søndergaard L, Mumtaz M, et al. Surgical or transcatheter aortic-valve replacement in intermediate-risk patients. N Engl J Med. 2017;376(14):1321-31.
13. Reardon MJ, Kleiman NS, Adams DH, Yakubov SJ, Coselli JS, Deeb GM, et al. Outcomes in the randomized CoreValve US Pivotal High Risk Trial in patients with a Society of Thoracic Surgeons risk score of $7 \%$ or less. JAMA Cardiol. 2016;1(8):945-9.

14. D'Agostino RS, Jacobs JP, Badhwar V, Fernandez FG, Paone G, Wormuth DW, et al. The Society of Thoracic Surgeons Adult Cardiac Surgery Database: 2019 update on outcomes and quality. Ann Thorac Surg. 2019;107(1):24-32.

15. Popma JJ, Deeb GM, Yakubov SJ, Mumtaz M, Gada H, O'Hair D, et al. Transcatheter aortic valve replacement with a self-expanding valve in low-risk patients. N Engl J Med. 2019;380(18):1706-1715.

ABSTRACT. Background: Cardiac valvulopathies occur in 1 of 100,000 inhabitants in Central America. Aortic stenosis represents $25 \%$ of these pathologies. The main cause is rheumatic fever and in high-income countries, congenital anomalies. Bicuspid aortic valve disease is a rare anomaly, present in $0.5 \%$ of the total population. Traditionally in Honduras, corrections of these anomalies had been made with vascular surgery. Description of the clinical cases: The first two cases of transcateter replacement or endovascular therapy (transcatheter aortic valve replacement, TAVR) performed in Honduras are presented, they were realized on 2019, in a private healthcare center in Tegucigalpa. The first case on a bivalve aorta, with the support of an In Snare system, due to the difficult approach, with EuroScore II $<4 \%$. The second case of an aortic stenosis secondary to rheumatic fever. Both procedures were performed without immediate or late complications. Echocardiography reported resolution of pressure gradients and reduction of maximum aortic transvalvular velocity (Vmax Ao). Conclusion: Being this a first-world therapy, indicated for patients with high and low surgical risk, it opens the opportunity for our population to receive an effective, safe therapy with immediate results.

Keywords. Aorta, Aortic diseases, Aortic valve stenosis, Transcatheter aortic valve replacement. 